\title{
Issues about Monitoring the Energy Performance of a PV Plants Constellation
}

\author{
S. Vergura \\ Dipartimento di Elettrotecnica ed Elettronica \\ Politecnico di Bari \\ Via E. Orabona 4, 70100 Bari (Italy) \\ Phone/Fax number:+39 080 5963590, e-mail: vergura@poliba.it
}

\begin{abstract}
After setting PhotoVoltaic (PV) plant up, it needs to monitor its energy performance in order to verify the correct or incorrect operation. For this aim, PV plants can be equipped with simple dataloggers or SCADA systems. When several PV plants have to be monitored, a unique supervision centre is implemented, even if each PV plant is independent from the others. From the point of view of storing and transferring data, they constitute a constellation of PV plants. This structure, based on the client-server configuration, allows to concentrate the information on a unique supervision centre, but PV plants have to be monitored one at a time. If several analyses have to be run for each PV plant, the total time for monitoring the whole constellation can be very long. The paper deals with these issues.
\end{abstract}

\section{Keywords}

PV plant, Constellation, Monitoring, Statistics.

\section{Introduction}

Italian PV market is very attractive for new gridconnected PV plants installations as well as for Operation and Maintenance (O\&M) enterprises, thanks to the government feed-in tariff. O\&M enterprises (usually related to the EPC contractors) can have a lot of PV plants to be monitored. Lately, PV plants was equipped with simple dataloggers, sometimes including ports to link directly the environmental sensors (radiance level, temperature and so on). Nowadays, large PV plants can be equipped with Supervisory Control And Data Acquisition (SCADA) systems. These systems implement not only the functions of the simple dataloggers but also other supervision functions. In fact, SCADA systems for PV plant can: a) reveal anomalies related to the breakers; b) measure the reactive power at the Point of Common Coupling (PCC); c) verify if connection pins of PV modules have been changed; d) other functions chosen by the end user, adding I/O ports. Some times SCADA system for monitoring PV plants are based on KNX standard in order to uniform the information acquired by different sensors. These complex systems allows to verify the total daily/monthly/yearly produced energy as well as the voltage and current waveform in both AC and DC sides. Nevertheless, sometimes operation anomalies are little and no warning is generated from the supervision system; even the evaluation of some standard benchmarks [1] can be ineffective. These hidden anomalies cause two problems: a) produced energy is less than the expected one, then economic revenue is reduced; b) anomalies become failures sooner or later. Paper [2] proposes a methodology to monitor the efficiency of a PV plant and to extract information about its operation. It allows to highlight anomalies also when standard benchmarks (defined in [1]) fail. Descriptive and inferential statistical tools are used in [2]. Also [3] consider statistics for assessing PV plants.

Moreover, when several PV plants have to be monitored, O\&M enterprises prefer to implement a unique supervision centre, directly linked to each PV plant. Each of them is analyzed one after the other. If few PV plants with small rated power have to be monitored, the whole monitoring system is effective and does not require too time, but when each PV plant has great rated power $(\geq 100 \mathrm{kWp}$ ), the amount of the data explodes and the processing time grows exponentially. The paper presents these issues in Sec. 2 and proposes possible solutions for an effective and efficient monitoring of a PV plants constellation in Sec 3. Results are reported in Sec. 4.

\section{Data to be monitored in a PV plants constellation}

This section deals with the issue of the great amount of data to be monitored for a PV plants constellation.

Fig. 1 reports the standard client-server configuration of several PV plants from the point of view of storing and transferring data. Obviously each PV plant has its own data acquisition system and they are independent each other from the electrical point of view. Server box is placed in a remote control room, while the data-logger (or SCADA system) of each PV plant is located near the plant. Sever box is linked to each data-logger by means of an Internet connection.

In order to implement an effective and efficient monitoring, data processing procedures have to be implemented. Even if the server box can manage sequentially the data of different plants, the whole 
processing time is high with respect to the need of a quick information about the operation of each PV plant.

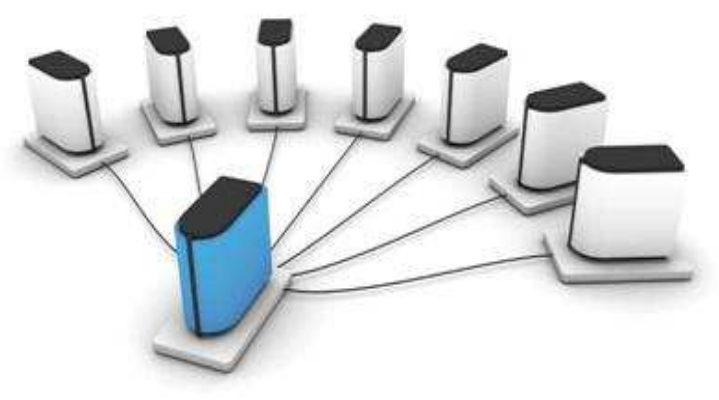

FIG. 1. CLIENT-SERVER CONFIGURATION FOR PV PLANT CONSTELLATION

As example, let us consider the amount of data to be processed for a constellation of ten $100-\mathrm{kWp}$ PV plants.

A single $100 \mathrm{kWp}$ rated power $\mathrm{PV}$ plant can be constituted by about 50 arrays. If the own data-logger samples each 10 minutes for 10 hour/day, 6 data set/hour and 60 dataset/day for each array have to be stored; then, 3.000 dataset/day for 50 arrays and 1.095 .000 data set/year for the whole PV plant. Each data set is usually constituted by several measures in DC side (voltage, current and power), several measures in $\mathrm{AC}$ side (voltage, current, power, energy) and several other parameters (cell temperature, environment temperature, radiance level and so on).

Even if the data processing procedure is based on only 4 variables as voltage, energy, cell temperature and radiance, 4.380.000 data for each PV plant have to be processed. The data to be processed for ten $100-\mathrm{kWp} \mathrm{PV}$ plants are about 43.800.000.

\section{Proposed Strategy}

The proposed strategy to monitor the energy performance of a PV plants constellation consists in carrying out preliminary analysis on each PV plant utilizing sampled data in role of the whole data population, in order to manage a smaller dataset for each PV plant. If an anomaly is revealed on a specific PV plant, a successive in depth analysis (based on the whole data population of that PV plant) will be carried out.

The sampling gives correct information if the sample is representative of the population. Sometimes, in order to obtain a representative sample, it is needed to consider more and more samplings and the final sample is obtained after processing the previous ones. Obviously, if the population is numerous, the total sampling time can be high for a prefixed significance level and the sampling advantage is lost, while the inaccuracy remains.

In the next Sections it will be shown that it is possible to utilize effectively the bootstrap technique[4-5] in role of a simple sampling. It will result that the bootstrap technique: a) allows to define a representative sample of the energy data stored in the data-logger of a PV plant; b) is more efficient than ordinary sampling technique, requiring a smaller computational time.
Moreover, another issue regards the time window of the analyzed data. In fact, different information are obtained if the analyzed data belong to a 1-month-based dataset or to yearly-based one. In fact the yearly-based dataset allows taking into account the variability of the environmental parameters for the whole year, but it is not effective to wait for a whole year to extract information about the operation of a PV plant. Then, a possibility to take into account all these issues consists in monitoring PV plants by means of cumulative statistical analyses [6]. This approach allows to get preliminary information about the operation of PV plants (even if small datasets are available) and to follow the trend of specific benchmarks during the whole year.

\section{Results}

Let us consider just a PV plants constellation as described in Sec. II, i.e. ten $100-\mathrm{kWp}$ PV plants. All the PV plants are located in southern Italy and are fixed, i.e. none of them has solar tracking system. The slope of the arrays of the ten PV plants ranges in $\left[30^{\circ} \div 44^{\circ}\right]$, while modules point to the south. Each single PV plant is constituted by identical modules, whereas different PV plants can contain different modules (mono-Silicon and poly-Silicon). For analogy, each single PV plant is constituted by identical DC-AC converters, but different $\mathrm{PV}$ plants are equipped by different configurations of DC-AC converters with respect to number of inverters and/or single rated power and/or technology.

The energy performance of the $10 \mathrm{PV}$ plants is evaluated in three ways, considering the following data: a) the whole data population; b) sampled data, obtained by 5 simple samplings on $20 \%$ of the population; c) resampled data, obtained by bootstrap technique with 5 resamplings on $20 \%$ of the population. Then, the previous three datasets are compared each other in order to verify the accuracy of the simple sampling and the bootstrap one with respect to the population, beside the computational times.

Particularly, following cases will be studied:

1) 1-month analysis (January 2009)

2) 3-months analysis (January-March 2009)

3) 6-months analysis (January-June 2009)

4) 12-months analysis (January-December 2009)

5) 12-months analysis (January-December 2009) based on 8 PV plants.

At the end of the last analysis, the trend of a specific benchmarks will be proposed.

\section{A. 1-month analysis (January 2009)}

Table I and Table II report the monthly-based results of the PV plants $1 \div 5$ and $6 \div 10$, respectively.

The first row of each table reports the number of PV plant under investigation, while the second one shows the mean values of the energy produced by each PV plant in one month (January 2009). These mean values are based on all the available data, then they are referred to the data populations. 
The third row reports the mean value of the previous mean values in order to calculate the mismatch of the energy produced by a single PV plant with respect the general mean value. This evaluation can give interesting information if the PV plant are quite similar each other with respect to the rated power and/or the components and/or the setup conditions (tilt, azimuth, and so on). Anyway, once the mismatches are calculated they can constitute relative operation benchmarks for the future. Then, fourth row reports the relative per cent mismatches between the mean energy value of each PV plant and the general mean energy value $\left(\mu_{g e n}\right)$. It can be noted that the maximum mismatches, in absolute value, regard the PV plants numbered $2(-3.39 \%)$ and $6(2.08 \%)$, respectively.

The second part of Table I and II reports the mean energy values of the PV plants based on the sample data, as previously said. These mean energy values are based on 5 simple samplings on $20 \%$ of the data population. Now, the spread in per cent, for each PV plant, represents the mismatch between the mean energy value based on the sampling data and the mean energy value based on the population data. It can be noted that the mismatches range in $[-4.11 \% \div 3.76 \%]$.

The third part of the Table I and II reports the mean energy values of the PV plants based on the bootstrapped data. These mean energy values are based on 5 resamples on $20 \%$ of the data population. The spread in per cent, for each PV plant, represents the mismatch between the mean energy value based on the bootstrapped data and the mean energy value based on the population data. It can be noted that the mismatches range in [$2.43 \% \div 1.40 \%$ ].

TAB. I. ENERGY VALUES (IN KWH) OF THE MEANS FOR POPULATIONS, SAMPLES, BOOTSTRAP RESAMPLES AND RELATIVE PER CENT SPREADS OF PV PLANTS $1 \div 5$ (1-MONTH)

RELATIVE PER CENT SPREADS OF PV PLANTS 1 5 (1-MONTH)
\begin{tabular}{|c|c|c|c|c|c|}
\hline & PV 1 & PV 2 & PV 3 & PV 4 & PV 5 \\
\hline$\mu_{\text {gen }}$ & 2760 & 2651 & 2744 & 2741 & 2787 \\
\hline$\%$ & 0.58 & -3.39 & 0 & -0.11 & 1.57 \\
\hline \multicolumn{5}{|c|}{2744} \\
\hline $\bar{x}$ & 2691 & 2699 & 2743 & 2721 & 2788 \\
\hline$\%$ & -2.49 & 1.81 & -0.03 & -0.72 & 0.04 \\
\hline \multicolumn{7}{|c|}{ Means with simple sampling and spreads \% } \\
\hline $\bar{x} *$ & 2752 & 2678 & 2747 & 2737 & 2787 \\
\hline$\%$ & -0.29 & 1.02 & 0.10 & -0.15 & 0 \\
\hline
\end{tabular}

TAB. II. ENERGY VALUES (IN KWH) OF THE MEANS FOR POPULATIONS, SAMPLES, BOOTSTRAP RESAMPLES AND RELATIVE PER CENT SPREADS OF PV PLANTS $6 \div 10$ (1-MONTH)

\begin{tabular}{|c|c|c|c|c|c|}
\hline & PV 6 & PV 7 & PV 8 & PV 9 & PV 10 \\
\hline$\mu$ & 2801 & 2769 & 2710 & 2689 & 2789 \\
\hline$\mu_{\text {gen }}$ & \multicolumn{5}{|c|}{2744} \\
\hline$\%$ & 2.08 & 0.91 & -1.24 & -2.00 & 1.64 \\
\hline \multicolumn{7}{|c|}{ Means with simple sampling and spreads \% } \\
\hline $\bar{x}$ & 2906 & 2718 & 2599 & 2671 & 2730 \\
\hline$\%$ & 3.76 & -1.83 & -4.11 & -0.66 & -2.09 \\
\hline \multicolumn{7}{|c|}{ Means with bootstrap and spreads \% } \\
\hline $\bar{x} *$ & 2869 & 2752 & 2706 & 2687 & 2750 \\
\hline$\%$ & 2.43 & -0.61 & -0.24 & -0.07 & -1.40 \\
\hline
\end{tabular}

For this analysis bootstrap technique results more effective than simple sampling, because the maximum spreads with respect to the populations are smaller.

Moreover, the total processing time for the three cases (population data, simple sampling and bootstrap) has been the following: a) 602 seconds; b) 217 seconds; c) 96 seconds. Then bootstrap results effective and efficient.

\section{B. 3-month analysis (January-March 2009)}

Table III and Table IV report the results, based on three months, of the PV plants $1 \div 5$ and $6 \div 10$, respectively. Also in this case the first parts of both the tables represent the population data, the second parts the simple samplings, while the third parts the bootstrapped ones. Again, the maximum mismatches with respect to the general mean energy value, in absolute value, regard the PV plants numbered $2(-2.79 \%)$ and 6 (3.91\%), respectively.

Analyzing the value obtained by simple samplings, it can be noted that all the mismatches belong to the range $[-3.71 \% \div 1.42 \%]$, similar to the previous case. PV plant numbered 6 has the smallest spread $(-3,71 \%)$, whereas in the previous case the same PV plant had the maximum spread $(3,76 \%)$.

For what concern the bootstrapped data, it results that all the spreads range in $[-1.65 \% \div 0.93 \%]$.

Moreover, the total processing time for the three cases (population data, simple sampling and bootstrap) has been the following: a) 4619 seconds; b) 1627 seconds; c) 698 seconds. Then, also for the 3-months analysis bootstrap technique has been effective and efficient.

TAB. III. ENERGY VALUES (IN KWH) OF THE MEANS FOR POPULATIONS, SAMPLES, BOOTSTRAP RESAMPLES AND RELATIVE PER CENT SPREADS OF PV PLANTS 1 $\div 5$ (3-MONTHS)

\begin{tabular}{|c|c|c|c|c|c|}
\hline & PV 1 & PV 2 & PV 3 & PV 4 & PV 5 \\
\hline$\mu$ & 20700 & 19983 & 20611 & 20578 & 20913 \\
\hline$\mu_{\text {gen }}$ & \multicolumn{5}{|c|}{20557} \\
\hline$\%$ & 0.70 & -2.79 & 0.26 & 0.10 & 1.73 \\
\hline \multicolumn{6}{|c|}{ Means with simple sampling and spreads \% } \\
\hline $\bar{x}$ & 20532 & 20021 & 20598 & 20345 & 20134 \\
\hline$\%$ & -0.81 & 0.19 & -0.06 & -1.13 & -3.72 \\
\hline \multicolumn{7}{|c|}{ Means with bootstrap and spreads \% } \\
\hline $\bar{x} *$ & 20612 & 20020 & 20650 & 20512 & 20567 \\
\hline$\%$ & -0.42 & 0.18 & 0.19 & -0.32 & -1.65 \\
\hline
\end{tabular}

TAB. IV. ENERGY VALUES (IN KWH) OF THE MEANS FOR POPULATIONS, SAMPLES, BOOTSTRAP RESAMPLES AND RELATIVE PER CENT SPREADS OF PV PLANTS $6 \div 10$ (3-MONTHS)

\begin{tabular}{|c|c|c|c|c|c|}
\hline & PV 6 & PV 7 & PV 8 & PV 9 & PV 10 \\
\hline$\mu$ & 20921 & 20738 & 20360 & 20145 & 20618 \\
\hline$\mu_{\text {gen }}$ & \multicolumn{5}{|c|}{20557} \\
\hline$\%$ & 3.91 & 0.83 & -0.07 & -1.40 & 1.32 \\
\hline \multicolumn{7}{|c|}{ Means with simple sampling and spreads \% } \\
\hline $\bar{x}$ & 20134 & 20912 & 20345 & 20432 & 20348 \\
\hline$\%$ & -3.71 & 0.84 & -0.07 & 1.42 & -1.3 \\
\hline \multicolumn{6}{|c|}{ Means with bootstrap and spreads \% } \\
\hline $\bar{x} *$ & 20674 & 20671 & 20456 & 20332 & 20548 \\
\hline$\%$ & -1.18 & -0.32 & 0.47 & 0.93 & -0.34 \\
\hline
\end{tabular}




\section{6-month analysis (January-June 2009)}

Table $\mathrm{V}$ and Table VI report the cumulative results, based on six months, of the PV plants $1 \div 5$ and $6 \div 10$, respectively. Also in this case the first parts of both the tables represent the population data, the second parts the simple samplings, while the third parts the bootstrapped ones. The maximum mismatches with respect to the general mean energy value, in absolute value, regard the PV plants numbered $2(-3.00 \%)$ and 6 (2.33\%), respectively. The trend of the spreads for these two PV plants are confirmed as new data are added. Then, the choices of the components (PV modules, inverters and so on) and the designing specifications (tilt, azimuth, and so on) are showing their effects. The next cumulative analysis, based on the data of a whole year, will confirm these trends.

Analyzing the value obtained by simple samplings, it can be noted that all the mismatches belong to the range $[-2.08 \% \div 3.23 \%$ ], similar to the two previous cases. PV plant numbered 1 has the smallest spread $(-2,08 \%)$, while PV plant numbered 3 has the maximum one $(3,23 \%)$.

For what concern the bootstrapped data, it results that all the spreads range in $[-1.13 \% \div 1.41 \%]$.

The total processing time for the three cases (population data, simple sampling and bootstrap) has been the following: a) 8948 seconds; b) 3313 seconds; c) 1523 seconds.

TAB. V. ENERGY VALUES (IN KWH) OF THE MEANS FOR POPULATIONS, SAMPLES, BOOTSTRAP RESAMPLES AND RELATIVE PER CENT SPREADS OF PV PLANTS $1 \div 5$ (6-MONTHS)

\begin{tabular}{|c|c|c|c|c|c|}
\hline & PV 1 & PV 2 & PV 3 & PV 4 & PV 5 \\
\hline$\mu$ & 71860 & 69174 & 71459 & 71437 & 72398 \\
\hline$\mu_{\text {gen }}$ & \multicolumn{5}{|c|}{71311} \\
\hline$\%$ & 0.77 & -3.00 & 0.21 & 0.18 & 1.52 \\
\hline \multicolumn{6}{|c|}{ Means with simple sampling and spreads \% } \\
\hline $\bar{x}$ & 70365 & 68800 & 73767 & 72944 & 73361 \\
\hline$\%$ & -2.08 & -0.54 & 3.23 & 2.1 & 1.33 \\
\hline \multicolumn{6}{|c|}{} \\
\hline $\bar{x} *$ & 72708 & 70149 & 70652 & 71587 & 71877 \\
\hline$\%$ & 1.18 & 1.41 & -1.13 & 0.21 & -0.72 \\
\hline
\end{tabular}

TAB. VI. ENERGY VALUES (IN KWH) OF THE MEANS FOR POPULATIONS, SAMPLES, BOOTSTRAP RESAMPLES AND RELATIVE PER CENT SPREADS OF PV PLANTS 6 $\div 10$ (6-MONTHS)

\begin{tabular}{|c|c|c|c|c|c|}
\hline & PV 6 & PV 7 & PV 8 & PV 9 & PV 10 \\
\hline$\mu$ & 72976 & 71992 & 70781 & 69936 & 71496 \\
\hline$\mu_{\text {gen }}$ & \multicolumn{5}{|c|}{71311} \\
\hline$\%$ & 2.33 & 0.96 & -0.74 & -1.93 & 0.26 \\
\hline \multicolumn{7}{|c|}{ Means with simple sampling and spreads \% } \\
\hline $\bar{x}$ & 71547 & 70617 & 69903 & 70908 & 71703 \\
\hline$\%$ & -1.38 & -1.91 & -1.24 & 1.39 & 0.29 \\
\hline \multicolumn{7}{|c|}{ Means with bootstrap and spreads \% } \\
\hline $\bar{x} *$ & 73316 & 72719 & 70951 & 69845 & 72204 \\
\hline$\%$ & 1.02 & 1.01 & 0.24 & -0.13 & 0.99 \\
\hline
\end{tabular}

Also for the 6-months analysis, bootstrap technique has been effective and efficient.

\section{12-month analysis (January-December 2009)}

Table VII and Table VIII report the cumulative results, based on a whole year, of the PV plants $1 \div 5$ and $6 \div 10$, respectively; the organization of the information follows the organization of the previous tables. Observing the table VIII, it can be noted that the spreads of the PV plants numbered 7 and 9 are particularly low. The energy produced by each one of these PV plants is very low and their values modify strongly the general mean energy value. An in depth investigation has allowed to know that these PV plants had out of order for maintenance for a very long time; then the first part of the tables is not reliable, because the numerousness of the available data is very different among the several PV plants.

Analyzing the value obtained by simple samplings, it can be noted that all the mismatches belong to the range $[-3.12 \% \div 3.09 \%]$, similar to the previous cases. PV plant numbered 6 has the smallest value $(-3,12 \%)$, while PV plant numbered 7 has the maximum one $(3,09 \%)$.

For what concern the bootstrapped data, it results that all the spreads range in $[-1.34 \% \div 1.20 \%]$.

Computational times have been the following: a) 15349 seconds; b) 6107 seconds; c) 2791 seconds.

TAB. VII. ENERGY VALUES (IN KWH) OF THE MEANS FOR POPULATIONS, SAMPLES, BOOTSTRAP RESAMPLES AND RELATIVE PER CENT SPREADS OF PV PLANTS $1 \div 5$ (12-MONTHS)

\begin{tabular}{|c|c|c|c|c|c|}
\hline & PV 1 & PV 2 & PV 3 & PV 4 & PV 5 \\
\hline$\mu$ & 143215 & 137213 & 142618 & 141835 & 143893 \\
\hline$\mu_{\text {gen }}$ & \multicolumn{5}{|c|}{138294} \\
\hline$\%$ & 3.56 & -0.78 & 3.13 & 2.56 & 4.13 \\
\hline \multicolumn{7}{|c|}{ Means with simple sampling and spreads \% } \\
\hline $\bar{x}$ & 146337 & 139133 & 145912 & 144841 & 146943 \\
\hline$\%$ & 2.18 & 1.40 & 2.31 & 2.12 & 2.12 \\
\hline \multicolumn{6}{|c|}{} \\
\hline $\bar{x} *$ & 144819 & 136746 & 141305 & 143423 & 144540 \\
\hline$\%$ & 1.12 & -0.34 & -0.92 & 1.12 & 0.45 \\
\hline
\end{tabular}

TAB. VIII. ENERGY VALUES (IN KWH) OF THE MEANS FOR POPULATIONS, SAMPLES, BOOTSTRAP RESAMPLES AND RELATIVE PER CENT SPREADS OF PV PLANTS 6 $\div 10$ (12-MONTHS)

\begin{tabular}{|c|c|c|c|c|c|}
\hline & PV 6 & PV 7 & PV 8 & PV 9 & PV 10 \\
\hline$\mu$ & 145052 & 123984 & 141263 & 119872 & 142997 \\
\hline$\mu_{\text {gen }}$ & \multicolumn{5}{|c|}{138294} \\
\hline$\%$ & 4.89 & -10.35 & 2.15 & -13.32 & 3.40 \\
\hline \multicolumn{6}{|c|}{ Means with simple sampling and spreads \% } \\
\hline $\bar{x}$ & 140526 & 127815 & 139271 & 117342 & 147271 \\
\hline$\%$ & -3.12 & 3.09 & -1.41 & -2.11 & 2.99 \\
\hline \multicolumn{6}{|c|}{} \\
\hline $\bar{x} *$ & 143108 & 124269 & 141799 & 118649 & 144712 \\
\hline$\%$ & -1.34 & 0.23 & 0.38 & -1.02 & 1.20 \\
\hline
\end{tabular}




\section{E. 12-month analysis (January-December 2009) based on 8 PV plants}

As in the previous case the energy produced by two PV plants has been very different from the others, this section reports the cumulative analysis based on eight PV plants; i.e. the PV plants numbered 7 and 9 have not been considered.

Then, Table IX and Table $X$ report the cumulative results, based on a whole year, of the PV plants $1 \div 5$ and 6-8-10, respectively. The organization of the information follows the organization of the previous tables. The maximum mismatches with respect to the general mean energy value, in absolute value, regard the PV plants numbered $2(-3.55 \%)$ and $6(1.96 \%)$, respectively. Considering only the eight well-working PV plants, it results that the trend of the spreads for these two PV plants is confirmed also for the yearly data. Analyzing the value obtained by simple samplings, it can be noted that all the mismatches belong to the range [$2.72 \% \div 3.82 \%$ ]. PV plant numbered 4 has the smallest spread $(-2,72 \%)$, while PV plant numbered 3 has the maximum one $(3,82 \%)$.

For what concern the bootstrapped data, it results that all the spreads range in $[-1.09 \% \div 1.02 \%]$.

Finally, computational times have been the following: a) 12152 seconds; b) 5839 seconds; c) 2512 seconds.

Once more time, bootstrap technique has been effective and efficient.

TAB. IX. ENERGY VALUES (IN KWH) OF THE MEANS FOR POPULATIONS, SAMPLES, BOOTSTRAP RESAMPLES AND

RELATIVE PER CENT SPREADS OF PV PLANTS 1 $\div 5$ (12-MONTHS)
\begin{tabular}{|c|c|c|c|c|c|}
\hline & PV 1 & PV 2 & PV 3 & PV 4 & PV 5 \\
\hline$\mu$ & 143215 & 137213 & 142618 & 141835 & 143893 \\
\hline$\mu_{\text {gen }}$ & \multicolumn{5}{|c|}{142261} \\
\hline$\%$ & 0.67 & -3.55 & 0.25 & -0.30 & 1.15 \\
\hline \multicolumn{7}{|c|}{ Means with simple sampling and spreads \% } \\
\hline $\bar{x}$ & 147382 & 134015 & 148066 & 137977 & 141720 \\
\hline$\%$ & 2.91 & -2.33 & 3.82 & -2.72 & -1.51 \\
\hline \multicolumn{6}{|c|}{} \\
\hline $\bar{x} *$ & 141635 & 136211 & 144072 & 142998 & 145259 \\
\hline$\%$ & -1.09 & -0.73 & 1.02 & 0.82 & 0.95 \\
\hline
\end{tabular}

TAB. X. ENERGY VALUES (IN KWH) OF THE MEANS FOR POPULATIONS, SAMPLES, BOOTSTRAP RESAMPLES AND RELATIVE PER CENT SPREADS OF PV PLANTS 6 $\div 10$ (12-MONTHS)

RELATIVE PER CENT SPREADS OF PV PLANTS 6 $\div 10$ (12-MONTHS)
\begin{tabular}{|c|c|c|c|c|c|}
\hline & PV 6 & PV 7 & PV 8 & PV 9 & PV 10 \\
\hline$\mu$ & 145052 & ----- & 141263 & ----- & 142997 \\
\hline$\mu_{\text {gen }}$ & \multicolumn{5}{|c|}{142261} \\
\hline$\%$ & 1.96 & ----- & -0.70 & ----- & 0.52 \\
\hline \multicolumn{7}{|c|}{ Means with simple sampling and spreads \% } \\
\hline $\bar{x}$ & 146023 & ---- & 145755 & ---- & 147186 \\
\hline$\%$ & 0.67 & ---- & 3.18 & ---- & 0.95 \\
\hline \multicolumn{7}{|c|}{ Means with bootstrap and spreads \% } \\
\hline $\bar{x} *$ & 145704 & ---- & 141517 & ---- & 144326 \\
\hline$\%$ & 0.45 & ---- & 0.18 & ---- & 0.93 \\
\hline
\end{tabular}

Finally, the mismatch values in per cent $\left(4^{\text {th }}\right.$ row of each table) between the energy produced by each PV plant and the general mean energy value are reported in Fig. 1 and 2. Particularly, Fig. 1 considers all the values of the cumulative analyses, then taking into account also the values of PV plants numbered 7 and 9 which have not produced energy for a very long time. Instead, Fig. 2 shows all the values of the cumulative analyses until PV plants numbered 7 and 9 have worked, whereas they have been not taken into account for the last yearly analysis, i.e. their values have been set to zero for the last analysis.

From Fig. 1, it can be observed that all the values are shut up between PV-2 (lower values) and PV-6 (higher values) for the first three analyses, then the values diverge because of the bad working of PV-7 and PV-9. Then, the trend of these benchmarks is able to give information about the correct operation of PV plants belonging to a constellation. In Fig. 2 (where the values of PV-7 and PV-9 have been set to zero for the last analysis), all the values belong to the channel defined by PV-2 and PV-6. Then, it is confirmed that PV-2 has the lower energy performance than the others, while PV-6 has the higher one. This information, based on the yearly data, was already extracted from the 1-month analysis.

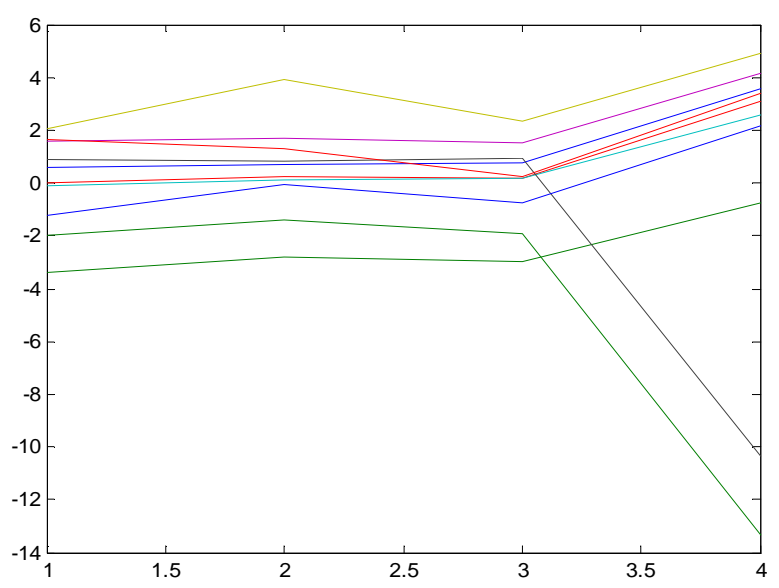

Fig. 1. TREND OF THE Mismatch VALUES FOR ALL THE PV PLANTS

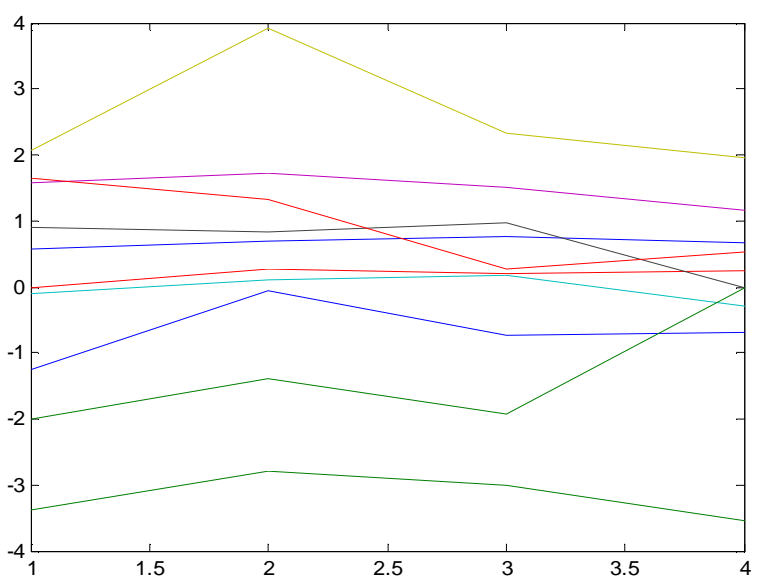

Fig. 2. TREND OF THE MISMATCH VALUES FOR ALL THE PV PLANTS UNITL PV-7 AND PV-9 HAVE WORKED, THENPV-7 AND PV-9 HAVE BEEN SET TO ZERO 


\section{Conclusions}

The paper proposes a systematic analysis about the energy performance of several PV plants belonging to a constellation. The paper is based on cumulative statistical analyses in order to verify the correct operation during the whole year. Experimental results will show five analyses in order to explain how the procedure is applied during a complete year, but it can be used for real-time monitoring. In this manner it is possible to follow the trend of the benchmarks and to characterize anomalies before they become failures. Nevertheless, cumulative analyses give no information about the typology of the anomaly and its cause.

The paper shows that the cumulative analysis is able to give preliminary information about the operation of the PV plants even if only partial data are available. In fact, the better operation of a specific PV plant has been already detected from the 1-month analysis. Moreover, the cumulative analysis is effective not only using the population data but also using the sampled data or bootstrapped ones. The paper shows also that bootstrap is effective in estimating the produced energy and efficient from the point of view of the computational time.
Finally, in my opinion an effective monitoring of a PV plants constellation has to determine which PV plant has to be analyzed for first. This is a very important task but it is not treated in this paper; it will be object of a future work.

\section{REFERENCES}

[1] CEI-IEC International Standard 61724- Photovoltaic system performance monitoring- Guidelines for measurement, data exchange and analysis, Ed. April 1998.

[2] S. Vergura, G. Acciani, V. Amoruso, G. Patrono, F. Vacca, "Descriptive and Inferential Statistics for Supervising and Monitoring the Operation of PV Plants", IEEE Trans on INDUSTRIAL Electronics, 2009, pp. 4456-4464.

[3] Paul D., Mukherjee D., Bhadra Ch audhuri S.R.,"Assessing solar PV behavior under varying environmental conditions- a statistical approach", in $4^{\text {th }}$ International Conference on Electrical and Computer Engineering ICECE 2006, 19-21 December 2006, Dhaka, Bangladesh.

[4] Efron, B., "Bootstrap Methods: Another Look at the Jackknife". The Annals of Statistics, 7 (1), pp 1-26, 1979.

[5] F. Vacca, S. Vergura, "Applying Bootstrap for Analyzing the Efficiency of PV Plants", ISIE 2010, July, 4-7, 2010, Bari, Italy, pp. 3311-3316 (ISBN 978-1-4244-6391-6).

[6] S. Vergura, "Cumulative Statistical Analysis to Monitor the Energy Performance of PV Plants", ICREPQ 2011, April, 13-15, 2011, Les Palmas de Gran Canaria, Spain. 